\title{
New Insights into the Fundamental Principle of Semiconductor Photocatalysis
}

\author{
Baoshun Liu,* Hao Wu, and Ivan P. Parkin
}

Cite This: ACS Omega 2020, 5, 14847-14856

Read Online

ABSTRACT: Although photocatalysis has been studied for many years as an attractive way to resolve energy and environmental problems, its principle still remains unclear. Some confusions and misunderstandings exist in photocatalytic studies. This research aims to elaborate some new thoughts on the fundamental principle of semiconductor photocatalysis. Starting from the basic laws of thermodynamics, we first defined the thermodynamic potential of photocatalysis. A concept, the Gibbs potential landscape, was thus
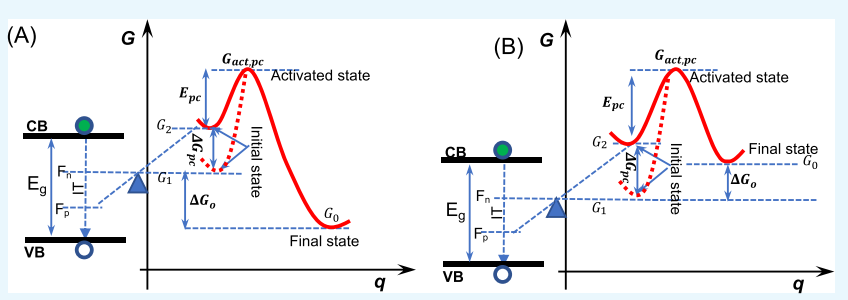
then proposed to describe the kinetics of photocatalysis. Photocatalysis is therefore defined as a light-driven chemical reaction that still needs heat activation, in that light and heat play their different roles and interact with each other. Photocatalysis should feature an activation energy functioning with both light and heat. The roles of light and heat are correlative and mutually inhibit at both levels of thermodynamics and kinetics, so it is impossible for an intrinsic light-heat synergism to happen. Two criteria were further proposed to determine an intrinsic light-heat synergism in photocatalysis. Experiments were also carried out to calculate the thermodynamic potential and can agree well with the theory. Experimental results proved that there is no intrinsic light-heat synergism, in accordance with our theoretical prediction. This research clarified some misunderstandings and gained some new insights into the nature of photocatalysis; this is important for the discipline of semiconductor photocatalysis.

\section{INTRODUCTION}

Photocatalysis helps address environmental and energy issues by using sunlight and has drawn much attention in the world over the last 50 years. $^{1-5}$ Different from classical thermocatalysis, its scientific nature depends on both light and heat. ${ }^{6-8}$ Although many efforts have been taken to study photocatalysis over decades, ${ }^{9-13}$ there still remain some confusions and misunderstandings on its principle, which must be clarified at deep and general levels. ${ }^{14-19}$

The kinetic processes involved in semiconductor photocatalysis are elaborated in Figure 1.,4,8 Light illumination excites the electronic transition from the valence band (VB) o the conduction band ( $\mathrm{CB}$ ) of a semiconductor; the generated electrons and holes then experience subsequent recombination and transfer to their acceptors. Figure 1B illustrates the band potentials, reduction potential of electron acceptors, and oxidation potential of hole acceptors. The more negative $\mathrm{CB}$ potential and more positive hole potential let the electrons and holes be capable of causing reduction and an oxidation effect, ${ }^{19}$ such as hydrogen and oxygen evolution from water. ${ }^{20}$ Figure 1 is the viewpoint that is popularly used to explain photocatalysis in literature.

Except for light excitation, the other processes are all dependent on temperature $(T)$ to some extent; for example, it has been revealed that the electron-hole recombination in $\mathrm{TiO}_{2}$ materials increases with temperatures because of the trapping effect; ${ }^{1,21,22}$ the transfer (processes 3 and 4 in Figure 1A) of electrons and holes to their acceptors, such as $\mathrm{O}_{2}$ and
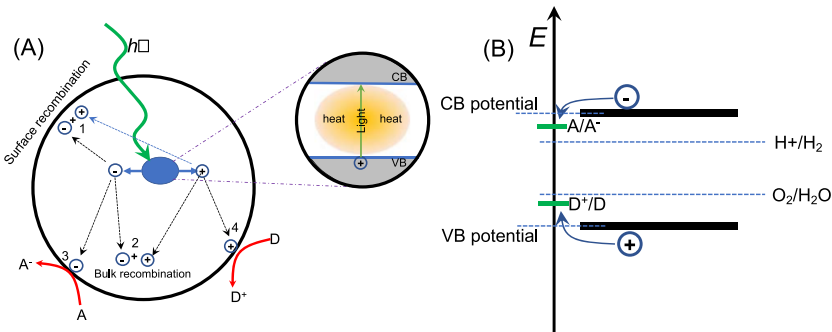

Figure 1. (A) Schematic of the kinetic processes of the charge carriers (holes and electrons) generated from the electronic transition from the valence band to the conduction band under light illumination. (1. Surface recombination; 2. Bulk recombination; 3. Electron transfer; 4. Hole transfer). The electron transfer and hole transfer are in direct connection with the photocatalytic effect. (B) Diagrammatic comparison between the band potentials (the conduction band potential and the valence band potential) and the reduction potential of an electron acceptor $\left(\mathrm{A} / \mathrm{A}^{-}\right)$and the oxidative potential of a hole acceptor $\left(\mathrm{D} / \mathrm{D}^{+}\right)$. The electric potentials of hydrogen evolution and oxygen evolution are also shown. The electrons and holes can be generated by light-induced electronic excitation; they can also be generated by heat via the lattice-electron interaction in a statistic way. $^{3,4,8}$

Received: May 8, 2020

Accepted: June 3, 2020

Published: June 15, 2020 


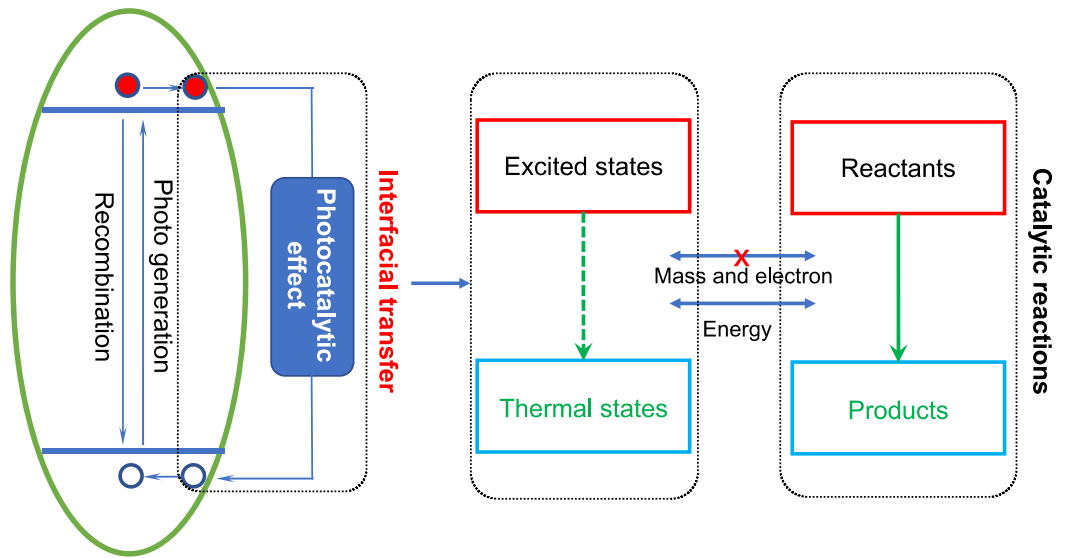

Figure 2. Relation between the interfacial transfer of electrons from the $\mathrm{CB}$ to the VB of a semiconductor and the induced photocatalytic effect.

water, was also found to be T-dependent. ${ }^{23-27} \mathrm{~A}$ number of studies have observed that the apparent kinetics of photocatalysis, including organic photocatalytic oxidations (PCO) and water photocatalytic splitting, feature heat activation. ${ }^{28,29}$ As the occurrence of photocatalysis needs heat assistance, photocatalysis has a thermodynamic potential.

The thermodynamic potential should be the most intrinsic thermodynamic problem for photocatalysis. Based on our understanding, the standpoint of Figure 1 might lead us to take the energy difference between the $\mathrm{CB}$ and VB potentials as the thermodynamic potential of photocatalysis. ${ }^{30,31}$ Heat can generate holes and electrons in a statistical way. ${ }^{32}$ As there is no difference in the absolute internal energies $(U)$ of the lightand heat-induced charge carriers, Figure $1 \mathrm{~B}$ also means that heat can drive the reactions happening in a photocatalytic way. For example, it has been thought that formaldehyde can be oxidized by heat-induced charge carriers in the dark; ${ }^{33}$ this however cannot agree with the second law of thermodynamics in that the $U$ cannot be the thermodynamic potential in an isothermal and isobaric system like photocatalysis. Therefore, what is the thermodynamic potential remains unclear and needs a clear elaboration.

Because of insufficient recognition of the thermodynamic potential, the intrinsic role of heat in photocatalysis is not well understood. Some common viewpoints might mistake the role of light with that of heat. Many publications said that light activates photocatalysis; it seems that photocatalysis does not need the assistance of heat; this is however in contrast to the experimental results that reported the existence of heat barriers in photocatalysis. $^{27-29}$ There are also some inconsistent studies on the role of heat. Some studies reported that heat can have a synergism with light in photocatalytic reactions. For example, Li et al. reported that heat can couple with light to increase the $\mathrm{PCO}$ of benzene over $\mathrm{TiO}_{2}$ at elevated temperatures. ${ }^{34}$ In addition to $\mathrm{TiO}_{2}$, such synergism was also proposed in the PCOs over other materials, such as $\mathrm{ZnO}$, $\mathrm{WO}_{3}$, and titanate. ${ }^{35-38}$ Many studies also attributed the increase of PCO rates at elevated temperatures to the normal activation of heat and did not claim a synergism. ${ }^{27-29,39}$ Whether and how heat can correlate with light in photocatalysis were not well elaborated in literature. The role of heat thus needs a fundamental clarification on a general level.

Starting from the laws of thermodynamics, the present research first defined the thermodynamic potential of semiconductor photocatalysis and elaborated the roles of heat and light in thermodynamics. A Gibbs free energy potential ( $G$ - potential) landscape was then proposed to illustrate the roles of light and heat as well as their correlation in kinetics. Based on the $G$-potential landscape, a heat activation model was advanced to describe the kinetics of photocatalysis. Photocatalysis is then defined as a light-driven chemical reaction activated by heat. It was clarified that it is impossible for light and heat to have a synergism because they are correlatively inhibited. Experiments were carried out to obtain the thermodynamic potential and compared with the theory. The effects of temperature and light intensity $(I)$ on photocatalysis were studied by experiments; this also showed good consistence with the theory. After ruling out a thermocatalytic contribution, some research studies meant that a light-heat synergism can be defined if the photocatalytic rates at elevated temperatures are higher than those at low temperatures. ${ }^{35-39}$ As photocatalysis contains heat processes that are dependent on temperatures in a Boltzmann statistical way, their definition to a light-heat synergism is inappropriate and will lead to misunderstandings. Two criteria were thus proposed to determine an intrinsic light-heat synergism in photocatalysis. The PCOs of acetone and formaldehyde over $\mathrm{TiO}_{2}$ were reexamined with the proposed criteria; this showed that the photocatalytic reactions do not involve a light-heat synergism, reconciling our theory with the studies that claimed the synergism.

The G-potential landscape in form unifies the thermodynamics and kinetics of photocatalysis to that of classical thermocatalysis. This allows us to think of photocatalysis in reference to thermal activation theory. It is thus hopeful that our ideas can deepen and widen the knowledge of photocatalytic science and help develop photocatalysis discipline.

\section{THEORETICAL ILLUSTRATION}

2.1. Roles of Light and Heat in Thermodynamics. Could heat drive photocatalysis? This question associates with the thermodynamic nature of photocatalysis. The standpoint of Figure 1 indicates that heat can also cause a catalytic effect similar to photocatalysis; this however conflicts with the second law of thermodynamics. The second law of thermodynamics requires that the $S$ (or the $U$ ) of an isolated system increases (decreases) to a maximum (or a minimum) until it reaches a thermodynamic equilibrium state in an irreversible process. Photocatalysis happens irreversibly under isothermal and isobaric conditions, so instead of the $S$ and $U$, the $G$ is its thermodynamic potential and tends to reduce itself 

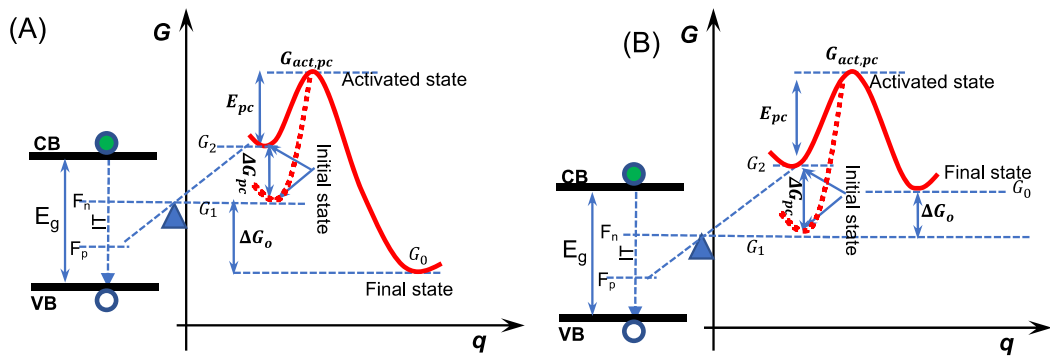

Figure 3. (A) Gibbs free energy landscape (G-potential landscape) of downhill photocatalytic reactions via the IT, such as PCOs; (B) G-potential landscape (G-potential landscape) of uphill photocatalytic reactions via the IT, such as the water splitting and the $\mathrm{CO}_{2}$ reduction.

until it reaches minimum at the equilibrium state. We will elaborate what is the $G$ and how it determines the principle of photocatalysis.

We discuss this topic by taking a semiconductor as a thermodynamic system. The photoinduced electronic transition lets a semiconductor change from thermodynamic equilibrium to nonequilibrium excited states. The excited state has a high $G$ position than the ground state because of the presence of the photoinduced charge carriers. The difference of their $G$ positions $\left(\Delta G_{\mathrm{IT}}\right)$ corresponds the quasi-Fermi level (quasi- $E_{\mathrm{F}}$ ) splitting ${ }^{32,40}$

$$
\Delta G_{\text {IT }}=-N_{\mathrm{A}}\left|F_{\mathrm{n}}-F_{\mathrm{p}}\right|=-N_{\mathrm{A}} E_{\mathrm{g}}+R T \ln \frac{N_{\mathrm{C}} N_{\mathrm{V}}}{\mathrm{np}}
$$

where $F_{\mathrm{n}}$ and $F_{\mathrm{p}}$ are the quasi-Fermi levels of the electrons and holes, $E_{\mathrm{g}}$ is the band gap, $T$ is the absolute temperature, $R$ is the idea gas constant, $\mathrm{n}$ and $\mathrm{p}$ are the densities of the electrons and holes, $N_{\mathrm{C}}$ and $N_{\mathrm{V}}$ are the effective densities of states at the $\mathrm{CB}$ and VB edges, $R$ is the ideal gas constant, and $N_{\mathrm{A}}$ is the Avogadro constant.

The $\Delta G_{\mathrm{IT}}$ becomes negative under light illumination; this drives the flow of the electrons from the $\mathrm{CB}$ to $\mathrm{VB}$ via the interfacial transfer (IT) that simultaneously induces a photocatalytic effect, as shown in Figure 2. It means that the $\Delta G_{\mathrm{IT}}$ is the thermodynamic driving force of photocatalysis, and the $G$ of the excited state of a semiconductor is the thermodynamic potential of photocatalysis. Therefore, it can be known that, although organic oxidations are downhill in thermodynamics, their occurrence in a photocatalytic way is not spontaneously allowed but needs a thermodynamic potential; this is different from thermocatalysis. Under an equilibrium state, $\Delta G_{\mathrm{IT}}$ becomes 0 according to the mass action law. The IT of electrons thus cannot happen in this case, as is photocatalysis. Therefore, heat cannot drive a reaction to happen in a photocatalytic way no matter how high the temperature is. The large difference between heat and light in their energy is not the intrinsic reason to distinguish their roles in photocatalysis, so the number of charge carriers do not determine the thermodynamics of photocatalysis. The attribution of dark catalysis to the heat-induced charge carriers is incorrect. ${ }^{24}$

Then, could heat affect the role of light in thermodynamics? We start from the first law of thermodynamics to discuss this question. The absolute inner energy $(\Delta U)$ of the electrons and holes can be transferred as a chemical work and heat dissipation to the environment according to eq 2 .

$$
\Delta U=\Delta A+\Delta Q
$$

We first discuss the reversible case; the first law of thermodynamics can be replaced with the Gibbs equation (the eq 3), which shows that the $\Delta G_{\mathrm{IT}}$ is less than the $\Delta U$ by an entropy term, $\mathrm{T} \Delta S$, representing the nonavailable energy (here, $\Delta U$ is the product of $N_{\mathrm{A}}$ and $E_{\mathrm{g}}$ of a semiconductor). ${ }^{32,40}$

$$
\Delta U=\Delta G_{\mathrm{IT}}+T \Delta S
$$

The comparison of eqs 1 and 3 shows that the $T \Delta S$ in the IT is 40

$$
T \Delta S=R T \ln \frac{N_{\mathrm{C}} N_{\mathrm{V}}}{\mathrm{np}}
$$

The statistical population of electrons and holes at the $\mathrm{CB}$ and VB states leads to the $T \Delta S$ term because the densities of photoinduced states only represent a small fraction of the densities of ground states. The $\Delta U$ includes the $\Delta G_{\text {IT }}$ and the $T \Delta S$. The $\Delta G_{\text {IT }}$ is the effective chemical work, and the $T \Delta S$ is a spontaneous heat loss. The above description took an assumption of a reversible process. Because photocatalysis is irreversible, the real chemical work $\left(\left|\Delta G_{\mathrm{pc}}\right|\right)$ that drives the electron IT and the photocatalytic effect is smaller than the $\left|\Delta G_{\mathrm{IT}}\right|$, and the real heat loss $\left(\Delta Q_{\mathrm{pc}}\right)$ happening in the IT is higher than the $T \Delta S$. Therefore, the first law of thermodynamics in photocatalysis is described with

$$
\Delta U=\Delta G_{\mathrm{pc}}+\Delta Q_{\mathrm{pc}}
$$

Because the $\mathrm{n}$ and $\mathrm{p}$ increase with the $I$, eq 4 shows that the $T$ and $I$ have opposite effects on the $T \Delta S$, so the $\Delta G_{\mathrm{IT}}$. It is reasonable to assume that the $T$ and $I$ have opposite effects on the $\Delta G_{\mathrm{pc}}$ and the $\Delta Q_{\mathrm{pc}}$. Therefore, the universal entropy increase principle leads to a mutual inhibition between light and heat in their thermodynamic roles, and a light-heat synergism cannot be defined in thermodynamics.

Thus, photocatalysis can be described from an energetic viewpoint. Figure 2 shows that the transfer of a semiconductor from the excited to the thermal-equilibrium states does not release electrons or mass; therefore, this in fact means that photocatalysis results from an energy transfer.

2.2. Roles of Heat and Light in Kinetics. As stated above, photocatalysis involves the generation of the charge carriers and the subsequent electron IT. The charge carrier generation is temperature-independent, but the electron IT is dependent on temperatures. By taking the irreversible feature into account, Figure 3 proposes the $G$-potential landscape of photocatalysis. The $\Delta G_{\mathrm{pc}}$ of eq 5 is transferred to the photocatalytic reactants and promotes their initial $G$ position (from $G_{1}$ to $G_{2}$ ); this opens new pathways for both downhill (Figure $3 \mathrm{~A}$ ) and uphill (Figure $3 \mathrm{~B}$ ) photocatalytic reactions. In the uphill cases, such as the water splitting and the $\mathrm{CO}_{2}$ reduction, when the $G_{2}$ is higher than the $G$ of the final state $\left(G_{0}\right)$, the uphill reactions become thermodynamically available, 
with part of the $\Delta G_{\mathrm{pc}}$ being stored in chemical bonds. ${ }^{41-43}$ However, in the downhill cases, such as PCOs, the initial $G$ position $\left(G_{1}\right)$ is not needed to be promoted to generate the thermodynamic driving force. It is considered that the energy transfer changes the pathways of organic oxidations by altering the heat activation of reactant species via the electron IT. How the $\Delta G_{\mathrm{pc}}$ is transferred to reactants relies on the detailed microscopic mechanism of electron IT; this is not the purpose of our study.

The initial state of photocatalysis includes the excited semiconductor and the photocatalytic reactants; the final state contains the thermal semiconductor and the photocatalytic products. The change in energy states of a semiconductor shows that photocatalysis cannot be classified as a conventional catalysis. ${ }^{44}$ A photocatalytic reaction via the electron IT (Figure 2) is written as

$$
\mathrm{SC}^{\text {illuminated }}+\text { reactants } \rightarrow \mathrm{SC}^{\text {Themal }}+\text { products }
$$

where $S C^{\text {illuminated }}$ and $S^{\text {Thermal }}$ denote the semiconductors at the excited and the thermal equilibrium states, respectively. Based on the Euler equation, the total $\Delta G$ is the sum of chemical potentials $\left(\mu_{i}\right)$ and particle number $\left(N_{i}\right)$ of all species. The $G$ of the final state is constant, while that of the initial state changes with the $G$ of the excited semiconductor.

The photocatalytic rate can be described by

$$
v \approx k \text { [reactants] }
$$

The roles of light and heat are enclosed in the apparent reaction rate constant $(k)$. The $k$ relates to temperatures in Arrhenius mode.

$$
k=k_{0} \exp -\frac{E_{\mathrm{pc}}}{R T}
$$

where $E_{\mathrm{pc}}$ is the real activation energy of photocatalysis. Statistically, $k_{0}$, the pre-exponential factor, is determined by thermal vibrations of reactants, which does not depend on light. Referring to the $G$-potential landscape, the $G$-difference between the $G_{\mathrm{act}, \mathrm{pc}}$ and the $G_{2}$ corresponds to the $E_{\mathrm{pc}}$. If a photocatalytic reaction is assumed to proceed in a reversible way, eq 8 can be changed to eq 9. The real $k$ of photocatalysis is smaller than eq 9 because of its irreversible feature.

$$
k=k_{0} \frac{\mathrm{np}}{N_{\mathrm{C}} N_{\mathrm{V}}} \exp \frac{-\left(G_{\mathrm{act}, \mathrm{pc}}-G_{1}-N_{\mathrm{A}} E_{\mathrm{g}}\right)}{R T}
$$

Equation 9 shows that $k$ is proportional to the $\mathrm{n}$ and $\mathrm{p}$, in line with the prediction of Figure 1. Based on the $G$-potential landscape and eq $1, E_{\mathrm{pc}}$ can be described as eq 10 in the reversible case. Although the real $E_{\mathrm{pc}}$ of a photocatalytic reaction is larger than eq 10 because of the irreversible feature, we can still use this formula to discuss the effects of $T$ and $I$ on the kinetics of photocatalysis.

$$
E_{\mathrm{pc}}(I, T)=G_{\mathrm{act}, \mathrm{pc}}-G_{1}-N_{\mathrm{A}} E_{\mathrm{g}}+R T \ln \frac{N_{\mathrm{C}} N_{\mathrm{V}}}{\mathrm{np}}
$$

It can be seen that the above description on the photocatalytic kinetics is the same as that of thermocatalysis. It should be noted that the experimentally derived activation energy $\left(E_{\text {act }}\right)$ is different from the $E_{\mathrm{pc}}$ because the last term of eq 10 is proportional to temperatures. The kinetics of photocatalysis is different from that of thermocatalysis because the $E_{\mathrm{pc}}$ decreases with the $I$ and increases with the $T$, so light and heat have inverse effects. An intrinsic light-heat synergism thus cannot be defined in the kinetics of photocatalysis. The Gpotential landscape shows that the photocatalytic activity can be increased by increasing the $G_{2}$ position and decreasing the $G_{\text {act,pc }}$ position (Figure 3). Inhibiting recombination or increasing light intensities increase the photocatalytic rates by upshifting the $G_{2}$ position; ${ }^{45,46}$ this does not affect the $E_{\text {act }}{ }^{47}$ Surface decorations with cocatalysts or defects might change the photocatalytic rates by lowering the $G_{\text {act,pc }}$ position, so the $E_{\text {act }}$ may be reduced. For example, we showed that $\mathrm{Cu}$ dopants can reduce the $E_{\text {act }}$ of acetone PCO over $\mathrm{TiO}_{2}{ }^{48}$ It is thus important to study the effect of surface decoration on the $E_{\text {act }}$ so as to have a deep understanding of the nature of photocatalysis.

Based on the above discussion, we thought that the usual statement of light activating photocatalysis might be misunderstood as to the role of light compared with that of heat. The Gpotential landscape indicates that this statement should be inappropriate as light solely cannot transfer reactants to activated states without heat assistance. This is in accordance with many experiments, as stated above. For example, the photocatalytic $\mathrm{CO}_{2}$ reduction and ethylene epoxidation are dependent on temperature. ${ }^{49,50}$ Our previous study and other first principle study further revealed that the electron IT to $\mathrm{O}_{2}$ include heat activation. ${ }^{27,47}$ It is thus considered by us that photocatalysis in principle is a reaction driven by light and activated by heat.

2.3. Rigorous Definition of a Light-Heat Synergism. The above illustration shows that light and heat are mutually inhibited. This is in contrast to some studies that reported a light-heat synergism at elevated temperatures. ${ }^{34-39}$ In many cases, the intermediates and final products (sometimes including carbonate or soot deposition) block active sites. Elevated temperatures help clean surfaces and might lead to an apparently meaningful light-heat synergism. In our opinion, this however cannot be taken as the intrinsic light-heat synergism, which could be confused in these studies. Therefore, the light-heat synergism should be carefully reexamined at a more rigorous level. In addition, we thought that the way used in defining a light-heat synergism is inappropriate in some studies. They considered that, by taking the thermocatalytic contribution out, a light-heat synergism can be obtained if the photocatalytic rates at elevated temperatures are higher than that at ambient temperatures. This will lead to an unrealistic result that almost all photocatalytic reactions involve a light-heat synergism because photocatalysis itself is heat-activated in principle. In any case, we cannot attribute the intrinsic role of heat to a heat-light synergism.

As we do not predict an intrinsic light-heat synergism in photocatalysis, the reconciliation of these results with our theory is needed. The number of particles participating in photocatalysis depends on heat and light in different manners. Heat activates reactants in the statistical way, so the number of activated particles depends on reciprocal temperature exponentially. Light affects the number of charge carriers sublinearly and linearly, depending on recombination types. Therefore, if heat has a synergism with light, the photocatalytic rates must be higher than that predicted by the Arrhenius formula, so the $T$-dependence of photocatalytic rates should bend upward in Arrhenius plots (Figure 4A, blue line). The downward-bending (Figure 4A, red line) and normal linear line (Figure 4A, black line) do not mean an intrinsic light-heat synergism. Similarly, if light has a synergism with heat, the 

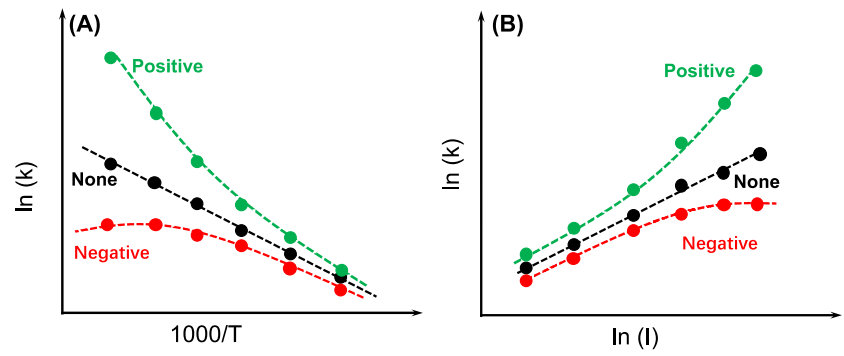

Figure 4. Kinetic diagram for determining the synergistic effect in semiconductor photocatalysis. (A) To determine the synergistic effect of heat on light; (B) to determine the synergistic effect of light on heat.

photocatalytic rates are higher than that predicted by a (sub-) linear mode in the $\log -\log$ plot, so the $I$-dependence of photocatalytic rates will bend upward with the I (Figure 4B, blue line). Other cases (black line and red line) do not mean an intrinsic light-heat synergism.

\section{EXPERIMENTAL VERIFICATION}

3.1. Experimental Determination of the $\Delta G_{\mathrm{IT}}$. The $\Delta G_{\text {IT }}$ is the thermodynamic driving force of photocatalysis. We will determine the $\Delta G_{\mathrm{IT}}$ of the PCOs of acetone and discuss its connection to the $I$ and the $T$. On-line electrical conductances were recorded to estimate the $\Delta G_{\mathrm{IT}}$. The electron transfer over PCOs is shown in Figure 5. ${ }^{51}$ Upon UV light illumination,

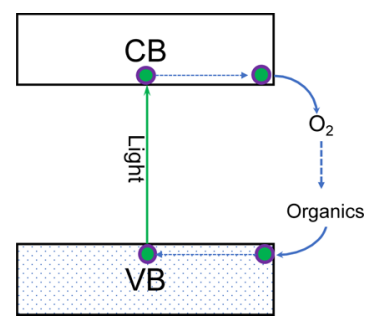

Figure 5. Schematic diagram of the electron IT pathway for organic PCO.

acetone molecules inject the electrons into the $\mathrm{VB}$ of $\mathrm{TiO}_{2}$, which are then promoted to the $\mathrm{CB}$ and transfer to $\mathrm{O}_{2}$. The kinetics of electron transfer to $\mathrm{O}_{2}$ is slower than that of hole injection, so the electrons accumulate in the $\mathrm{CB}$ and contribute to the conductances. Because the holes are quickly consumed by acetone, the $\Delta G_{\mathrm{IT}}$ is mainly contributed by the electrons according to eq 11 .

$$
\Delta G_{\mathrm{IT}}=-N_{\mathrm{A}}\left|F_{n}-F_{n_{0}}\right|=-R T \ln \frac{n}{n_{0}}
$$

where $F_{n_{0}}$ denotes the dark Fermi level of electrons. As the conductances are proportional to the density of electrons, this can be written as

$$
\Delta G_{\mathrm{IT}}=-R T \ln \frac{\sigma(I)}{\sigma(0)}
$$

where $\sigma(I)$ and $\sigma(0)$ are the photoconductance and the dark conductance.

The effect of the light intensities and temperatures on the electric conductances and the acetone conversions $(\alpha)$ are shown in Figures $\mathrm{S} 1-\mathrm{S} 4$, respectively. Figure $6 \mathrm{~A}$ shows the dependences of the $\Delta G_{\mathrm{IT}}$ and the $\alpha$ on the light intensities, which show a correlation between them. Figure $6 \mathrm{~B}$ shows the dependences of the $\Delta G_{\mathrm{IT}}$ and the $\alpha$ with temperatures. This shows a different result that the decrease in the $\Delta G_{\text {IT }}$ accompanies an increase in the $\alpha$. The increase of the $\Delta G_{\mathrm{IT}}$ with the light intensities is attributed to the increased number of electrons; this can naturally increase the photocatalytic activity. Because the electron transfer to $\mathrm{O}_{2}$ increases with temperatures, ${ }^{52,53}$ so the density of electrons decreases with temperatures. The $\Delta G_{\mathrm{IT}}$ accordingly decreases with temperatures according to eq 9. However, the decrease in the $\Delta G_{\mathrm{IT}}$ does not mean a decrease in photocatalytic activity, because the increase in temperatures also increases heat activation. Therefore, it is seen that the increases of the light intensities and the temperatures have inverse effects on the $\Delta G_{\mathrm{IT}}$, so the $E_{\mathrm{pc}}$ changes inversely with respect to the light intensities and temperatures; this agrees well with our theoretical prediction.

3.2. Comparison between the Experimental Photocatalytic Rates and That Predicted from the Theory. The relative change of the $E_{\mathrm{pc}}$ on the light intensities and the temperatures can be calculated from the electric conductances, based on which the relative changes in photocatalytic rates can be estimated from eq 6. By referring to the $G$-potential landscape, the photocatalytic rates at different light intensities are related to the photoconductances according to eq 13 .

$$
r(I)=\frac{k(I)}{k\left(I_{0}\right)}=\mathrm{e}^{G_{2}(I)-G_{2}\left(I_{0}\right) / R T}=\frac{\sigma(I)}{\sigma\left(I_{0}\right)}
$$

where $k(I), k\left(I_{0}\right), \sigma(I)$, and $\sigma\left(I_{0}\right)$ denote the photocatalytic rate constants and the on-line photoconductances at the light intensity of $I$ and $I_{0}$, respectively. $I_{0}$ is set as the reference $I$. The photocatalytic rates at different temperatures depend on the on-line conductances according to eq 14 .
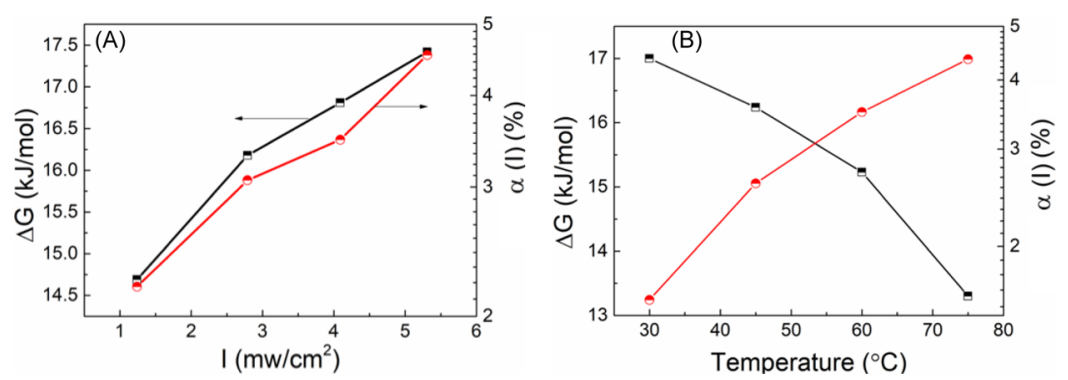

Figure 6. (A) Linear dependence of the $\Delta G_{\mathrm{IT}}$ and semi-log dependence of the acetone conversions on the light intensities (A) and temperatures (B). 

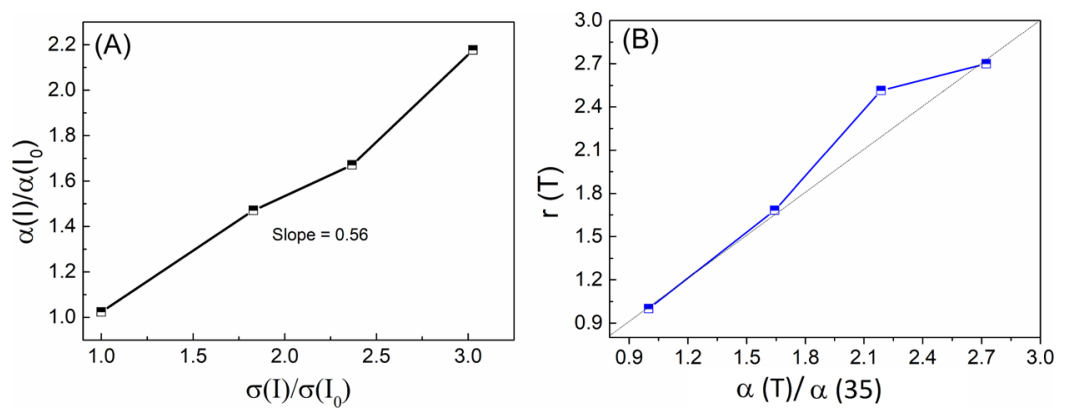

Figure 7. Correlation between the experimental-derived acetone photocatalytic conversions in the case of different light intensities (A) and temperatures (B).
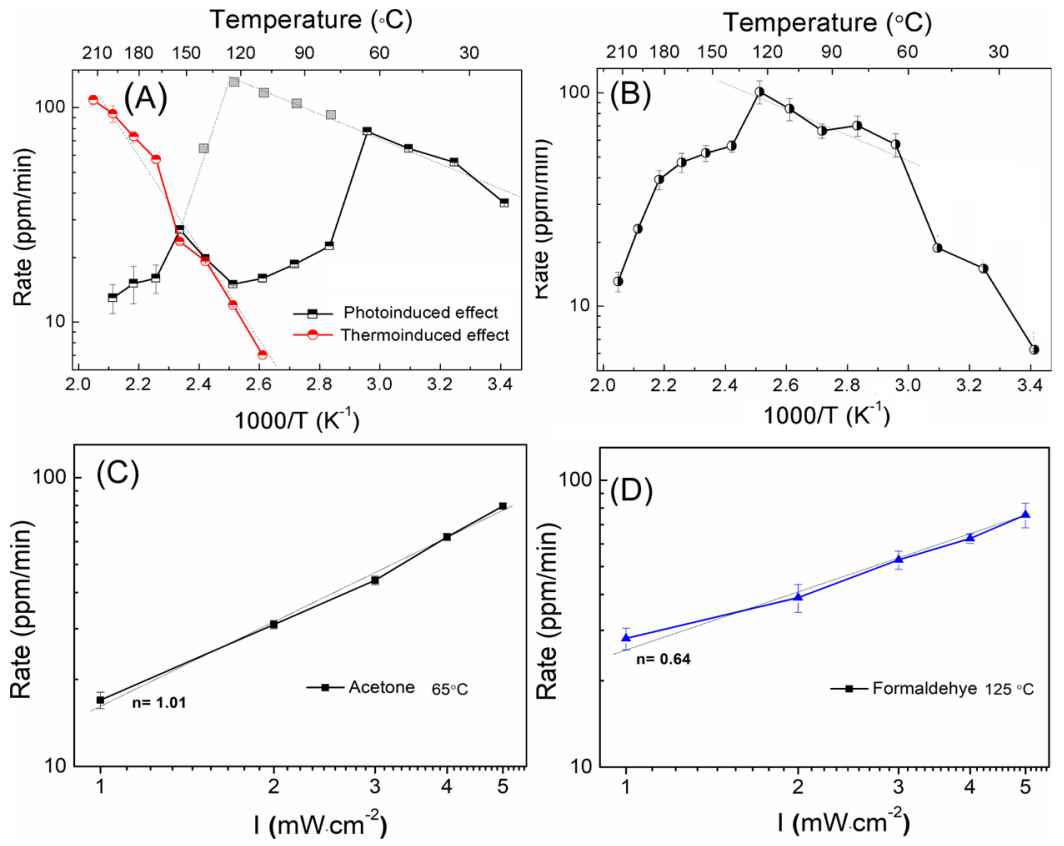

Figure 8. (A) Arrhenius plots of the PCOs and thermocatalytic oxidations of acetone by $\mathrm{TiO}_{2}$ at different temperatures; (B) Arrhenius plot of the photocatalysis of the PCOs of formaldehyde over $\mathrm{TiO}_{2}$ at different temperatures; (C) log-log dependence of the acetone PCO rates on light intensities (experiments were done at $65^{\circ} \mathrm{C}$ ); (D) $\log -\log$ dependence of the formaldehyde PCO rates on light intensities (the experiments were done at $\left.125^{\circ} \mathrm{C}\right)$.

$$
r(T)=\frac{k(T)}{k\left(T_{0}\right)}=\mathrm{e}^{-E_{0}\left(\frac{1}{k T_{1}}-\frac{1}{k T_{0}}\right)} \frac{\sigma(T)}{\sigma\left(T_{0}\right)}
$$

where $k(T), k\left(T_{0}\right), \sigma(T)$, and $\sigma\left(T_{0}\right)$ denote the photocatalytic rate constants and photoconductances at the temperatures of $T$ and $T_{0}$, respectively. $T_{0}$ is set as the reference temperature, and $E_{0}$ is a constant.

The $I_{0}$ and the $T_{0}$ were set as $7.6 \mathrm{~mW} / \mathrm{cm}^{2}$ and $40{ }^{\circ} \mathrm{C}$, respectively. We compared the correlations between the experimentally derived $\alpha$ and that estimated from eqs 13 and 14, as shown in Figure 7A,B, respectively. Figure 7A shows a close linear correlation that supports the role of light in increasing the photocatalytic activity by decreasing the $E_{\mathrm{pc}}$. The line slope is 0.56 , smaller than 1 as predicted in eq 13 . The electrons first transfer to $\mathrm{O}_{2}$ and then contribute to the PCO of acetone. Figure $7 \mathrm{~A}$ means that the electron transfer to $\mathrm{O}_{2}$ is faster than that contributing to photocatalysis. Our previous study showed that the electron transfer to $\mathrm{O}_{2}$ contributes to the photoassisted $\mathrm{O}_{2}$-sorption recombination. ${ }^{44}$ This might lead to a fast change of photoconductances compared to the photocatalytic activity. In addition, the assumed kinetic model is based on the reversible case that cannot fully accord with the real case. The photocatalytic rate predicted from a reversible model is naturally higher than the real irreversible case, in good accordance with the experimental results. It was reported that some intermediated products, including acetate and formate, can be formed during the PCOs of acetaldehyde. ${ }^{13}$ We thought that similar products might also be formed in the course of acetone PCOs over $\mathrm{TiO}_{2}$; this may block the active sites and lead to a decrease in photocatalytic rates as compared to the theoretical prediction, as shown in Figure 7A.

Eq 14 shows that the relation between experimental-derived $\alpha$ and $r(T)$ is more complicated. The term $\exp \left(-E_{0}(1 / k T-\right.$ $\left.\left.1 / k T_{0}\right)\right)$ is the function of heat activation; the term $\sigma(T) /$ $\sigma\left(T_{0}\right)$ is the effect of temperatures on the $\Delta G_{\text {IT }}$. It is seen from Figure $6 \mathrm{~B}$ that the $\Delta G_{\mathrm{IT}}$ decreases with temperatures, so the increase of acetone conversion with temperature is due to the role of heat activation. Figure $7 \mathrm{~B}$ shows the correlation between the $r(T)$ and $\sigma(T) / \sigma\left(T_{0}\right)$ by setting the $E_{0}$ at $55 \mathrm{~kJ} /$ mol. According to Figure 3 , the $E_{0}$ corresponds to the difference between $G_{\text {act,pc }}$ and $G_{1}$. The prediction of eq 14 can accord with experiments to some extent. However, eq 14 does not predict a pure Arrhenius mode as the experimentally 
derived $\sigma(T) / \sigma\left(T_{0}\right)$ varied with temperatures; this shows that the predicted photocatalytic rates tend to decrease at elevated temperatures. Because the electron transfer is dependent on the temperatures, the electron density $(n)$ in $\mathrm{TiO}_{2}$ is also affected by the temperatures in addition to light intensities. Our theory predicts the $T$-dependence of photocatalytic rates on the premise that $\mathrm{n}$ is independent of temperatures. However, it is inevitable for $n$ to change with temperatures. The increase of electron transfer results in a decrease in $n$ with temperatures. Therefore, the change of the $\Delta G_{\mathrm{IT}}$ indicated from $\sigma(T) / \sigma\left(T_{0}\right)$ is larger than that predicted from our theory, which assumes that $n$ does not change with temperatures. This finally leads to the result that the photocatalytic rates predicted from eq 14 deviate from the experimental results.

3.3. Reconciliation of the Reported Light-Heat Synergism to Our Theory. Temperatures were first varied to show whether heat can have a synergism with light in the PCOs of acetone over $\mathrm{TiO}_{2}$. The catalytic rates were obtained from $\mathrm{CO}_{2}$ evolutions at different temperatures in the presence and absence of light illuminations (Figures S5 and S6). As acetone can also be oxidized in the dark, pure photocatalytic rates were obtained by subtracting the total rates with that in the dark. Figure $8 \mathrm{~A}$ shows the Arrhenius plots of the thermocatalysis (red line) and the photocatalysis (black line). The thermocatalytic oxidations agree well with Arrhenius mode, and the apparent activation energy $\left(E_{\text {app }}\right)$ is $42 \mathrm{~kJ} / \mathrm{mol}$. The PCOs also show an Arrhenius dependence on temperatures below $65{ }^{\circ} \mathrm{C}$, with an $E_{\text {app }}$ of $8.0 \mathrm{~kJ} / \mathrm{mol}$. At the temperatures ranging between 65 and $140{ }^{\circ} \mathrm{C}$, the photocatalytic rates show a first-decrease-then-increase because of the carbonate deposit and the removal by thermocatalysis. The photocatalytic effect starts to decrease again and goes on to disappear when the temperatures are higher than $140{ }^{\circ} \mathrm{C}$, possibly because of the inhibition of heat on the role of light. The fictitious photocatalytic rates were also plotted after excluding the effect of carbonate deposit. Despite the effect of carbonate deposit, the results still shows that the photocatalytic rates first increase and then decrease with temperatures. Figure $8 \mathrm{~A}$ shows that the PCOs of acetone do not locate in the positive region of Figure $4 \mathrm{~A}$, and thus heat cannot have a synergism with light. Light intensities were also varied to determine whether light can have a synergism with heat. Figure $8 \mathrm{~B}$ shows the log-log dependence of the photocatalytic rates on the light intensities. The index $n$ is about $1\left(\right.$ rate $\propto I^{n}$ ), indicating that the PCOs of acetone should belong to a singleelectron-driven process. The log-log plot does not locate in the positive region of Figure $4 \mathrm{~B}$, so light cannot produce a synergism with heat in the acetone photocatalysis.

Formaldehyde PCOs were also used to check an intrinsic light-heat synergism. $\mathrm{CO}_{2}$ evolutions at different temperatures in the presence and absence of light illuminations are shown in Figures S7 and S8. Figure 8B,D shows the dependences of the photocatalytic rates on the temperatures and light intensities in Arrhenius and $\log -\log$ modes, respectively. Formaldehyde was not subject to the thermocatalytic oxidations at all temperatures, and there is no carbonate that can deposit on the $\mathrm{TiO}_{2}$ surfaces. The photocatalytic rates show a first-increase-thendecrease with temperatures, and depend on the light intensities in the sublinear mode $(n=0.64)$. The photocatalytic rates below $65{ }^{\circ} \mathrm{C}$ are lower than the Arrhenius predictions because of inadequate evaporation of formaldehyde aqueous solutions. The PCOs between 65 and $125{ }^{\circ} \mathrm{C}$ agree well with Arrhenius mode; the $E_{\text {app }}$ is about $9.0 \mathrm{~kJ} / \mathrm{mol}$. The photocatalytic rates show a continuous decrease and go on to disappear when the temperatures are higher than $125^{\circ} \mathrm{C}$, also possibly due to the light-heat coinhibited effect. The PCOs do not locate in the positive regions of Figure $4 \mathrm{~A}, \mathrm{~B}$. It has also been reported that formaldehyde PCOs over $\mathrm{TiO}_{2}$ show a decrease in the $n$ at high light intensities. ${ }^{54}$ It is concluded that the formaldehyde PCOs should not include a light-heat synergism.

Some studies reported the location of PCOs in the positive region of Figure $4 \mathrm{~A} .{ }^{55,56}$ We considered that the results are hardy to be believed because the observed superexponential behaviors are completely beyond the theory of statistical thermodynamics. We thought that other effects should be carefully taken out before confirming an intrinsic light-heat synergism. For example, if light-induced surface heating is underestimated, the measured photocatalytic rates can go to the "positive" region of Figure 4A. In recent studies, we found that the light-induced increase of surface temperatures is always underdetermined. Therefore, in a broad sense, although their results might be attributed to a "light-heat synergism", it does not mean the intrinsic one defined above.

The experimental results are in accordance with our theory, so an intrinsic light-heat synergism cannot exist in the PCOs over $\mathrm{TiO}_{2}$, which is opposite to the conclusions obtained in some studies. ${ }^{34,57,58}$ According to these works, if the pure photocatalytic rates at elevated temperatures are higher than that at ambient temperature, a light-heat synergism can be defined. Based on this rule, a similar conclusion can be obtained that the PCO of acetone at $65{ }^{\circ} \mathrm{C}$ and the PCO of formaldehyde at $125{ }^{\circ} \mathrm{C}$ should have a light-heat synergism because their rates are higher than that at ambient temperature. We think that these works might take the natural increase of photocatalytic rates with temperatures as a lightheat synergism. Because the photocatalysis itself contains heatactivated processes, we cannot attribute such results to a lightheat synergism essentially. The light-heat synergism is a concept that relates to the fundamentals of photocatalytic science, so great care must be taken to study light-heat synergism in photocatalysis so as to avoid misunderstandings. It is therefore better to use our proposed criteria to provide a rigorous definition.

\section{CONCLUSIONS}

A detailed elaboration was given on the fundamental principle of semiconductor photocatalysis on both thermodynamics and kinetics levels. The $G$ of the charge carriers in semiconductors was defined as the thermodynamic potential of photocatalysis. Heat is incapable of driving catalysis happening in a photocatalytic way. A G-potential landscape of photocatalysis was also proposed. The role of light in kinetics is included in the apparent activation energy. In addition to the activation, heat can also affect the apparent activation energy. Photocatalysis was thus defined as the light-driven chemical reaction activated by heat, in that the roles of heat and light also correlate with each other. We obtained the conclusion that there is no intrinsic light-heat synergism in semiconductor photocatalysis as light and heat mutually inhibit at both the thermodynamic and kinetic levels. Rigorous criteria were further proposed to reconcile the light-heat synergism reported in some studies to our theory. Experimental results agree well with the theory. We clarified some confusions and misunderstandings on the fundamentals of semiconductor photocatalysis. The above proposed theory should be 
important for photocatalytic discipline and open a door for thinking of photocatalysis from an energetic perspective.

\section{EXPERIMENTAL DESCRIPTION}

5.1. On-Line Electrical Conductances. On-line electric conductances over the acetone photocatalysis were measured in a self-designed flow bed quartz reactor system (refer to our recent publication $\left.{ }^{39}\right)$. Pure $\mathrm{N}_{2}(5 \mathrm{~N})$, pure $\mathrm{O}_{2}(5 \mathrm{~N})$, and acetone $-\mathrm{N}_{2}$ mixture $\left(500 \mathrm{ppm}\right.$ of acetone in pure $\mathrm{N}_{2}$ ) were continuously let into the reactor under careful control by flow meters. The flow rates of $\mathrm{N}_{2}, \mathrm{O}_{2}$, and acetone- $\mathrm{N}_{2}$ gases were set as $1,0.05$, and $0.01 \mathrm{~L} / \mathrm{min}$, respectively. The concentrations of acetone in the outlet of the reactor were monitored by a gas chromatograph equipped with a FIB detector (Shimadzu GC2500). A thin $\mathrm{TiO}_{2}$ coating was coated on a piece of quartz glass substrate by doctor-blading from $\mathrm{p} 25$ paste; this was then annealed at $450{ }^{\circ} \mathrm{C}$ for $1 \mathrm{~h}$. Two gold electrodes were deposited on the $\mathrm{TiO}_{2}$ coating, with a $0.2 \mathrm{~mm}$ gap being left for conductance measurements. Two larger area $\mathrm{TiO}_{2}$-coated quartz glasses were also prepared for acetone oxidations. A heat plate was used to heat the reactor to the set temperature, which was monitored by a PT100 resistance temperature detector. A mercury lamp (USHIO SP-9) equipped with a 365 $\mathrm{nm}$ band-passed optical filter was used as the light source. The intensity of the light reaching the $\mathrm{TiO}_{2}$ surface was determined by a $\mathrm{Si}$ diode photodetector. Photocatalytic activity was evaluated by acetone conversation $(\alpha)$, defined as the ratio of the difference between the acetone concentrations before and after UV light illumination. The on-line electron conductances were simultaneously monitored by an electric source meter (KEITHLEY 2450 Source meter).

5.2. Catalytic Experiments. Photocatalytic and thermocatalytic experiments were done in a self-designed quartz closed-circulation cylindrical reactor (refer to our recent publication ${ }^{43}$ ). The reactor was linked with a photoacoustic IR multigas monitor (INNOVA Air Tech Instruments model 1412). The reactor was heated to the set temperature before catalytic experiments. The same light source was used, and the light intensity was checked by a $\mathrm{Si}$ diode photodetector. Infrared thermography (FLIR E60) was used to confirm that the light illumination had no effect on surface temperatures. As carbonate contaminations can also lead to $\mathrm{CO}_{2}$ evolution, all samples were pretreated by UV light before photocatalysis reactions. Fresh air was then flowed through the reactor to purge out the old air until the residual $\mathrm{CO}_{2}$ concentration was lower than $20 \mathrm{ppm}$.

Acetone and formaldehyde were used for the PCOs. Two experiments were designed for photocatalysis and thermocatalysis. The reactor was kept in the dark for $60 \mathrm{~min}$ at the set temperature, and after that, $2 \mu \mathrm{L}$ of liquid acetone or formaldehyde was injected into the reactor to see a pure thermocatalytic effect. The photocatalytic effect was measured according to the same procedure under simultaneous light illumination. The $\mathrm{CO}_{2}$ evolution rates were used to evaluate photocatalytic activity. Pure photocatalytic effects were obtained by subtracting the $\mathrm{CO}_{2}$ evolutions under light illumination from that in the dark.

\section{ASSOCIATED CONTENT}

\section{s) Supporting Information}

The Supporting Information is available free of charge at https://pubs.acs.org/doi/10.1021/acsomega.0c02145.
Time dependences of on-line conductances and photocatalytic effects in the courses of the PCOs of acetone and formaldehyde over $\mathrm{TiO}_{2}$ materials at different temperatures and light intensities and time dependences of the $\mathrm{CO}$ evolutions during the PCOs acetone and formaldehyde over $\mathrm{TiO} 2$ at different temperatures (PDF)

\section{AUTHOR INFORMATION}

\section{Corresponding Author}

Baoshun Liu - State Key Laboratory of Silicate Materials for Architectures, Wuhan University of Technology, Wuhan City, Hubei Province 430070, People's Republic of China; 이이.org/0000-0001-5564-3685; Email: liubaoshun@ 126.com

\section{Authors}

Hao Wu - State Key Laboratory of Silicate Materials for Architectures, Wuhan University of Technology, Wuhan City, Hubei Province 430070, People's Republic of China

Ivan P. Parkin - Department of Chemistry, Materials Chemistry Centre, University College London, London WC1H OAJ, U.K.

Complete contact information is available at:

https://pubs.acs.org/10.1021/acsomega.0c02145

\section{Notes}

The authors declare no competing financial interest.

\section{ACKNOWLEDGMENTS}

B.L. thanks the National Natural Science Foundation of China (nos. 51772230 and 51461135004), the Hubei Foreign Science and Technology cooperation project (2017AHB059), and the 101 project (no. B18038). I. P. Parkin thanks the Wuhan University of Technology for a visiting professorship. B.L. also thanks the Open Foundation of Key Laboratory for UV-Emitting Materials and Technology of Ministry of Education, Northeast Normal University (grant nos. 130028908 and 135130013). We thank Dr Daibing Luo from Analytical@Testing Center of Sichuan University for his help in structural analysis.

\section{REFERENCES}

(1) Liu, B.; Zhao, X.; Yu, J.; Parkin, I. P.; Fujishima, A.; Nakata, K. Intrinsic Intermediate Gap States of $\mathrm{TiO}_{2}$ Materials and Their Roles in Charge Carrier Kinetics. J. Photochem. Photobio. C: Photochem. Rev. 2019, 39, 1-57.

(2) Fujishima, A.; Zhang, X.; Tryk, D. $\mathrm{TiO}_{2}$ Photocatalysis and Related Surface Phenomena. Surf. Sci. Rep. 2008, 63, 515-582.

(3) Schneider, J.; Matsuoka, M.; Takeuchi, M.; Zhang, J.; Horiuchi, Y.; Anpo, M.; Bahnemann, D. W. Understanding $\mathrm{TiO}_{2}$ Photocatalysis: Mechanisms and Materials. Chem. Rev. 2014, 114, 9919-9986.

(4) Sun, X.; Huang, H.; Zhao, Q.; Ma, T.; Wang, L. Thin-Layered Photocatalysts. Adv. Funct. Mater. 2020, 30, 1910005.

(5) Lin, S.; Zhang, Y.; You, Y.; Zeng, X.; Ma, T.; Huang, H. Bifunctional Hydrogen Production and Storage on 0D-1D Heterojunction of $\mathrm{Cd}_{0.5} \mathrm{Zn}_{0.5} \mathrm{~S} @$ Halloysites. Adv. Funct. Mater. 2019, 29, 1903825.

(6) Fujishima, A.; Rao, T. N.; Tryk, D. A. Titanium Dioxide Photocatalysis. J. Photochem. Photobio. C: Photochem. Rev. 2000, 1, 121.

(7) Nakata, K.; Fujishima, A. $\mathrm{TiO}_{2}$ Photocatalysis: Design and Applications. J. Photochem. Photobio. C: Photochem. Rev. 2012, 13, 169-189. 
(8) Linsebigler, A. L.; Lu, G.; Yates, J. T. Photocatalysis on $\mathrm{TiO}_{2}$ Surfaces: Principles, Mechanisms, and Selected Results. Chem. Rev. 1995, 95, 735-758.

(9) Low, J.; Cheng, B.; Yu, J. Surface Modification and Enhanced Photocatalytic $\mathrm{CO}_{2}$ Reduction Performance of $\mathrm{TiO}_{2}$ : a Review. Appl. Surf. Sci. 2017, 392, 658-686.

(10) Liu, B.; Cheng, K.; Nie, S.; Zhao, X.; Yu, H.; Yu, J.; Fujishima, A.; Nakata, K. Ice-water Quenching Induced $\mathrm{Ti}^{3+}$ Self-doped $\mathrm{TiO}_{2}$ with Surface Lattice Distortion and the Increased Photocatalytic Activity. J. Phys. Chem. C 2017, 121, 19836-19848.

(11) Chen, X.; Liu, L.; Huang, F. Black Titanium Dioxide $\left(\mathrm{TiO}_{2}\right)$ Nanomaterials. Chem. Soc. Rev. 2015, 44, 1861-1885.

(12) Li, X.; Yu, J.; Jaroniec, M.; Chen, X. Cocatalysts for Selective Photoreduction of $\mathrm{CO}_{2}$ into Solar Fuels. Chem. Rev. 2019, 119, $3962-4179$.

(13) Melchers, S.; Schneider, J.; Emeline, A.; Bahnemann, D. Effect of $\mathrm{H}_{2} \mathrm{O}$ and $\mathrm{O}_{2}$ on the Adsorption and Degradation of Acetaldehyde on Anatase Surfaces-An In Situ ATR-FTIR Study. Catalysts 2018, 8, 417.

(14) Wang, X.; Zeng, D.; Xie, C. Temperature-dependent Photothermocatalytic Mineralization of Gaseous Benzene Based on Rutile $\mathrm{TiO}_{2}$ Nanorod Aarray. Mater. Lett. 2015, 139, 36-339.

(15) Lan, L.; Li, Y.; Zeng, M.; Mao, M.; Ren, L.; Yang, Y.; Liu, H.; Yun, L.; Zhao, X. Efficient UV-vis-infrared Light-driven Catalytic Abatement of Benzene on Amorphous Manganese Oxide Supported on Anatase $\mathrm{TiO}_{2}$ Nanosheet with Dominant $\{001\}$ Facets Promoted by a Photothermocatalytic Synergetic Effect. Appl. Catal. B: Environ. 2017, 203, 494-504.

(16) Xie, W.; Li, Y.; Shi, W.; Zhao, L.; Zhao, X.; Fang, P.; Zheng, F.; Wang, S. Novel effect of Significant Enhancement of Gas-phase Photocatalytic Efficiency for Nano ZnO. Chem. Eng. J. 2012, 213, 218-224.

(17) Li, Y.; Wang, C.; Zheng, H.; Wan, F.; Yu, F.; Zhang, X.; Liu, Y. Surface Oxygen Vacancies on $\mathrm{WO}_{3}$ Contributed to Enhanced Photothermosynergistic Effect. Appl. Surf. Sci. 2017, 391, 654-661.

(18) Peng, X.; Wang, C.; Li, Y.; Ma, H.; Yu, F.; Che, G.; Yan, J.; Zhang, X.; Liu, Y. Revisiting Cocatalyst $/ \mathrm{TiO}_{2}$ Photocatalyst in Blue Light Photothermalcatalysis. Catal. Today 2019, 335, 286-293.

(19) Low, J.; Jiang, C.; Cheng, B.; Wageh, S.; Al-Ghamdi, A. A.; Yu, J. A Review of Direct Z-Scheme Photocatalysts. Small Methods 2017, 1,1700080 .

(20) Hisatomi, T.; Kubota, J.; Domen, K. Recent Advances in Semiconductors for Photocatalytic and Photoelectrochemical Water Splitting. Chem. Soc. Rev. 2014, 43, 7520-7535.

(21) Stevanovic, A.; Yates, J. T., Jr. Electron Hopping through $\mathrm{TiO}_{2}$ Powder: a study by Photoluminescence Spectroscopy. J. Phys. Chem. C 2013, 117, 24189-24195.

(22) Yang, S.; Brant, A. T.; Giles, N. C.; Halliburton, L. E. Intrinsic Small Polarons in Rutile $\mathrm{TiO}_{2}$. Phys. Rev. B: Condens. Matter Mater. Phys. 2013, 87, 125201.

(23) Yoshihara, T.; Katoh, R.; Furube, A.; Tamaki, Y.; Murai, M.; Hara, K.; Murata, S.; Arakawa, H.; Tachiya, M. Identification of Reactive Species in Photoexcited Nanocrystalline $\mathrm{TiO}_{2}$ films by Widewavelength-range (400-2500 nm) Transient Absorption Spectroscopy. J. Phys. Chem. B 2004, 108, 3817-3823.

(24) Elbanna, O.; Fujitsuka, M.; Kim, S.; Majima, T. Charge Carrier Dynamics in $\mathrm{TiO}_{2}$ Mesocrystals with Oxygen Vacancies for Photocatalytic Hydrogen Generation under Solar Light Irradiation. J. Phys. Chem. C 2018, 122, 15163-15170.

(25) Liu, B.; Yang, J.; Zhao, X.; Yu, J. The Role of Electron Interfacial Transfer in Mesoporous nano- $\mathrm{TiO}_{2}$ Photocatalysis: a Combined Study of In Situ Photoconductivity and Numerical Kinetic Simulation. Phys. Chem. Chem. Phys. 2017, 19, 8866.

(26) Li, Y.-F.; Selloni, A. Theoretical Study of Interfacial Electron Transfer from Reduced Anatase $\mathrm{TiO}_{2}(101)$ to Adsorbed $\mathrm{O}_{2}$. J. Am. Chem. Soc. 2013, 135, 9195-9199.

(27) Hofstadler, K.; Bauer, R.; Novalic, S.; Heisler, G. New Reactor Design for Photocatalytic Wastewater Treatment with $\mathrm{TiO}_{2}$
Immobilized on Fused-Silica Glass Fibers: Photomineralization of 4Chlorophenol. Environ. Sci. Technol. 1994, 28, 670-674.

(28) Mills, A.; Morris, S. Photomineralization of 4-Chlorophenol Sensitized by Titanium Dioxide: a Study of the Initial Kinetics of Carbon Dioxide Photogeneration. J. Photochem. Photobiol., A 1993, $71,75-83$.

(29) Al-Sayyed, G.; D’Oliveira, J.-C.; Pichat, P. Semiconductorsensitized Photodegradation of 4-Chlorophenol in Water. J. Photochem. Photobiol., A 1991, 58, 99-114.

(30) Wen, J.; Li, X.; Liu, W.; Fang, Y.; Xie, J.; Xu, Y. Photocatalysis Fundamentals and Surface Modification of $\mathrm{TiO}_{2}$ Nanomaterials. Chin. J. Catal. 2015, 36, 2049-2070.

(31) Spasiano, D.; Marotta, R.; Malato, S.; Fernandez-Ibañez, P.; Di Somma, I. Solar photocatalysis: Materials, Reactors, Some Commercial, and Pre-industrialized Applications. A Comprehensive Approach. Appl. Catal. B: Environ. 2015, 170-171, 90-123.

(32) Liu, B.; Zhao, X.; Terashima, C.; Fujishima, A.; Nakata, K. Thermodynamic and Kinetic Analysis of Heterogeneous Photocatalysis for Semiconductor Systems. Phys. Chem. Chem. Phys. 2014, 16, 8751-8760.

(33) Yang, X.; Cao, C.; Hohn, K.; Erickson, L.; Maghirang, R.; Hamal, D.; Klabunde, K. Highly Visible-light Active C- and V-doped $\mathrm{TiO}_{2}$ for Degradation of Acetaldehyde. J. Catal. 2007, 252, 296-302.

(34) Li, Y.; Huang, J.; Peng, T.; Xu, J.; Zhao, X. Photothermocatalytic Synergetic Effect Leads to High Efficient Detoxification of Benzene on $\mathrm{TiO} 2$ and $\mathrm{Pt} / \mathrm{TiO} 2$ Nanocomposite. Chem CatChem 2010, 2, 1082-1087.

(35) Xie, W.; Li, Y.; Shi, W.; Zhao, L.; Zhao, X.; Fang, P.; Zheng, F.; Wang, S. Novel Effect of Significant Enhancement of Gas-Phase Photocatalytic Efficiency for Nano ZnO. Chem. Eng. J. 2012, 213, 218-224.

(36) Peng, X.; Wang, C.; Li, Y.; Ma, H.; Yu, F.; Che, G.; Yan, J.; Zhang, X.; Liu, Y. Revisiting Cocatalyst/ $/ \mathrm{TiO}_{2}$ Photocatalyst in Blue Light Photothermalcatalysis. Catal. Today 2019, 335, 286-293.

(37) Liu, Y.; Shu, W.; Chen, K.; Peng, Z.; Chen, W. Enhanced Photothermocatalytic Synergetic Activity Toward Gaseous Benzene for Mo+C-Codoped Titanate Nanobelts. ACS Catal. 2012, 2, 25572565.

(38) Li, Y.; Wang, C.; Zheng, H.; Wan, F.; Yu, F.; Zhang, X.; Liu, Y. Surface oxygen vacancies on WO 3 contributed to enhanced photothermo-synergistic effect. Appl. Surf. Sci. 2017, 391, 654-661.

(39) Matthews, R. W. Photooxidation of Organic Impurities in Water Using Thin Films of Titanium Dioxide. J. Phys. Chem. 1987, 91, 3328-3333.

(40) Salvador, P. Semiconductors' Photoelectrochemistry: A Kinetic and Thermodynamic Analysis in the Light of Equilibrium and Nonequilibrium Models. J. Phys. Chem. B 2001, 105, 6128-6141.

(41) Maeda, K.; Domen, K. Photocatalytic Water Splitting: Recent Progress and Future Challenges. J. Phys. Chem. Lett. 2010, 1, 26552661.

(42) Kudo, A.; Miseki, Y. Heterogeneous Photocatalyst materials for water splitting. Chem. Soc. Rev. 2009, 38, 253-278.

(43) Habisreutinger, S. N.; Schmidt-Mende, L.; Stolarczyk, J. K. Photocatalytic Reduction of $\mathrm{CO}_{2}$ on $\mathrm{TiO}_{2}$ and Other Semiconductors. Angew. Chem., Int. Ed. 2013, 52, 7372-7408.

(44) Ohtani, B. Revisiting the Fundamental Physical Chemistry in Heterogeneous Photocatalysis: its Thermodynamics and Kinetics. Phys. Chem. Chem. Phys. 2014, 16, 1788-1797.

(45) Park, H.; Kim, H.-i.; Moon, G.-h.; Choi, W. Photoinduced Charge Transfer Processes in Solar Photocatalysis Based on Modified $\mathrm{TiO}_{2}$. Energy Environ. Sci. 2016, 9, 411-433.

(46) Park, Y.; Kim, W.; Monllor-Satoca, D.; Tachikawa, T.; Majima, T.; Choi, W. Role of Interparticle Charge Transfers in Agglomerated Photocatalyst Nanoparticles: Demonstration in Aqueous Suspension of Dye-Sensitized $\mathrm{TiO}_{2}$. J. Phys. Chem. Lett. 2013, 4, 189-194.

(47) Liu, B.; Yang, J.; Zhao, X.; Yu, J. The Role of Electron Interfacial Transfer in Mesoporous nano- $\mathrm{TiO}_{2}$ Photocatalysis: a Combined Study of In Situ Photoconductivity and Numerical Kinetic Simulation. Phys. Chem. Chem. Phys. 2017, 19, 8866. 
(48) Unpublished results. We found that $\mathrm{Cu}$ dopants change the $E_{\text {act }}$ of photocatalytic organic oxidations over $\mathrm{TiO}_{2}$ by altering the $E$.

(49) Christopher, P.; Xin, H.; Marimuthu, A.; Linic, S. Singular Characteristics and Unique Chemical Bond Activation Mechanisms of Photocatalytic Reactions on Plasmonic Nanostructures. Nat. Mater. 2012, 11, 1044-1050.

(50) Zhang, X.; Li, X.; Zhang, D.; Su, N. Q.; Yang, W.; Everitt, H. O.; Liu, J. Product Selectivity in Plasmonic Photocatalysis for Carbon Dioxide Hydrogenation. Nat. Commun. 2017, 8, 14542.

(51) Liu, B.; Wang, X.; Wen, L.; Zhao, X. Investigation of Electron Behavior in $\mathrm{Nano}^{-\mathrm{TiO}_{2}}$ Photocatalysis by Using In Situ Open-Circuit Voltage and Photoconductivity Measurements. Chem.-Eur. J. 2013, 19, 10751-10759.

(52) Liu, B.; Wu, H.; Zhang, X.; Parkin, I. P.; Zhao, X. New Insight into the Role of Electron Transfer to $\mathrm{O}_{2}$ in Photocatalytic Oxidations of Acetone over $\mathrm{TiO}_{2}$ and the Effect of Au Co-catalyst. J. Phys. Chem. C 2019, 123, 30958-30971.

(53) Liu, B.; Wu, H.; Parkin, I. P. Gaseous Photocatalytic Oxidation of Formic Acid over $\mathrm{TiO}_{2}$ : A Comparison between the Charge Carrier Transfer and Light-Assisted Mars-van Krevelen Pathways. J. Phys. Chem. C 2019, 123, 22261-22272.

(54) Zhu, X.; Chang, D.-L.; Li, X.-S.; Sun, Z.-G.; Deng, X.-Q.; Zhu, A.-M. Inherent Rate Constants and Humidity Impact Factors of Anatase $\mathrm{TiO}_{2}$ Film in Photocatalytic Removal of Formaldehyde from Air. Chem. Eng. J. 2015, 279, 897-903.

(55) Jiang, D.; Wang, W.; Gao, E.; Zhang, L.; Sun, S. BismuthInduced Integration of Solar Energy Conversion with Synergistic Low-Temperature Catalysis in $\mathrm{Ce}_{1-\mathrm{x}} \mathrm{BixO}_{2-\delta}$ Nanorods. J. Phys. Chem. C 2013, 117, 24242-24249.

(56) Jiang, D.; Wang, W.; Zhang, L.; Qiu, R.; Sun, S.; Zheng, Y. A Strategy for Improving Deactivation of Catalytic Combustion at Low Temperature via Synergistic Photocatalysis. Appl. Catal. B: Environ. 2015, 165, 399-407.

(57) Liu, Y.; Shu, W.; Chen, K.; Peng, Z.; Chen, W. Enhanced Photothermocatalytic Synergetic Activity Toward Gaseous Benzene for Mo+C-Codoped Titanate Nanobelts. ACS Catal. 2012, 2, 25572565.

(58) Li, Y.; Wang, C.; Zheng, H.; Wan, F.; Yu, F.; Zhang, X.; Liu, Y. Surface Oxygen Vacancies on $\mathrm{WO}_{3}$ Contributed to Enhanced Photothermosynergistic Effect. Appl. Surf. Sci. 2017, 391, 654-661. 